\title{
A Review of Effects of Covid-19 on Pregnant Women and Neonates
}

\author{
Monika Adhau and Swaroopa Chakole \\ Department of Community Medicine, Jawaharlal Nehru Medical College, Datta Meghe Institute of Medical \\ Sciences (Deemed to be University), Sawangi (Meghe), Wardha-442001, Maharashtra, India \\ Corresponding author email: drswaroopachakole@gmail.com
}

\section{ABSTRACT}

Starting from Wuhan City of China, COVID-19pandemicreached the peak and invaded even the remote rural areas of the developing countries in a very short time. The transmission potential of this virus is massive which putundue burden on thehealthcare machinery and public health sector in a short period of time. The vulnerable sections of the society such as comorbid, elderly and pregnant women are on high risk of contracting the infection and developing severe symptoms of the disease that certainly needs medical attention on intensive care unit level. Pregnant women are more vulnerable than others because they harbor two lives within them and are already in immunosuppressive state. Various clinics and hospitals dedicated to nursing facility for pregnant women are being dedicated to COVID-19 treatment and thus creating difficulty for pregnant women to access the health care facility which they need in regular intervals. Infected pregnant women may transmit the virus to the offspring through vertical transmission and through post birth transmission.Dedicated health care facility for deliveries and post-natal care can be solution to minimize the maternal mortality rate and infant mortality rate due to COVID-19. Widespread authentic information needs to be disbursed in order to deal with the pandemic. Vulnerable groups such as pregnant women and neonates needs special attention to overcome the trauma physically as well as mentally to lead a happy life.

\section{KEY WORDS: PREGNANT WOMEN, PREGNANCY, VERTICAL TRANSMISSION, COVID-19, MATERNAL MORTALITY.}

\section{INTRODUCTION}

The news which everyone wants to hear is about vaccine of COVID-19. As the name suggests the coronavirus disease 2019 shortened as COVID-19 is the deadly pandemic which has been unfolded in past century. ( Dushyant et al., 2020) The emergence of the disease can be traced to the Wuhan city of the Hubei province of the China. From there it spreads rapidly and grew exponentially in all other parts of the world. So much so that World Health Organization (WHO) was compelled to declare it as pandemic(2)after seeing its widespread coverage and deadly outcome and done only one time now since its inception after second World War. The

Biosc Biotech Res Comm P-ISSN: 0974-6455 E-ISSN: 2321-4007

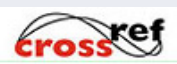

Identifiers and Pagination

Year: 2021 Vol: 14 No (6) Special Issue

Pages: $158-163$

This is an open access article under Creative

Commons License Attribn 4.0 Intl (CC-BY).

DOI: $h$ ttp://dx.doi.org/10.21786/bbrc/14.6.35 crown shaped strain of the coronavirus is new hence named novel coronavirus has been extremely uncertain and difficult to predict. Symptoms (Azer, 2020)have constantly changed from mild cough, cold, fever to loss of test and smell, diarrhea and acute respiratory distress syndrome (ARDS).

The previous outbreaks of severe acute respiratory syndrome (SARS) and Middle Eastern respiratory syndrome (MERS) were confined to particular part of the world but theCOVID-19 encompasses the whole world. Initial response of the scientific and governing agencies was of shock and were taken aback due to its rapid transmissibility and deadlier characteristics which requires hospitalization. The health care infrastructure (Sheriff, 2020)were crumbled due to enormous pressure that were put on them and were not capable to handle such pressure. Whole facility wasdedicated to contain the COVID-19 spread yet were and are facing difficulties. Major chunk of the resource pool is diverted to the containment of the COVID-19 and vaccine demand is on high so as to get rid of the situation. The vulnerable sections of the society such as comorbid (Wang et al.,

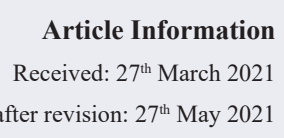

ccepted after revision: $27^{\text {th }}$ May 2021 
2020), aged people along with pregnant women are on high risk of contracting the infection and developing severe symptoms of the disease. Which may certainly need medical attention on intensive care unit level. Pregnant women are more vulnerable than others because they harbor two lives within them and are already in immunosuppressive state.

Various clinics and hospitals dedicated to nursing facility for pregnant women are being dedicated to COVID-19 treatment and thus creating difficulty for pregnant women to access the health care facility which they need in regular intervals. Infected pregnant women may transmit the virus to the offspring through vertical transmission and through post birth transmission. Former is constantly being debated as the evidence of the same is lacking nut the later have obvious reasons of human negligence while handling the infant while regular essential activities such as breastfeeding. Even a wrong sneeze can infect the baby if it is not already infected. The preterm birth in infected supposed mothersaw rise and can create complications. Still birth, death in one week of the delivery of the baby are the worst outcomes of the COVID-19 on pregnant women. Psychological distress is common in pregnant women and is aggravating due to uncertain situations around the world. The fear of transmitting the virus to the infant is most common and needs to be addressed. In this article the topics are reviewed and analyzed in accordance with different studies published across the world.

Covid-19 and Prenatal Stage: Coronavirus disease 2019 or COVID-19 causes due to novel coronavirus which was never identified before in humans. The novelty of the virus is so much that it is hard to design any containment plan since no historical evidence of virus's behavior is present with the scientists. It by far one year since the pandemic struck and proven deadly, one thing is established is that certain groups of the people are more vulnerable than others. Those includes elderlies with comorbidities, pregnant women's etc. which generally are going through their immunosuppressive state (Di et al., 2020). Pregnant women especially are more vulnerable and are placed in high-risk category since they involve two life's at risk. During pregnant state women are already vulnerable to various diseases and needs to be clinically monitored in regular times.

Now after the deadly virus has been proven hazardous the need of extra attention with proper caution is added in order to prevent the pregnant women from contracting the infection. Starting from diagnostics mechanism available there several ways to detect the pregnant women whether she is harboring the novel coronavirus or not. It includes a computed tomography scan of the chest or CT scan of the chest of the pregnant women, RT-PCR or reverse transcript polymerase chain reaction which is currently being used worldwide and antibodies test which detects certain types of the antibodies which are associated with COVID-19. Although CT scan of chest can infer some conclusion but it is not recommended to rely on its result as there are some flaws in the test. Antibodies test are the test detecting antibodies in the blood samples of the person to be tested and points towards whether that person has contracted the COVID-19 or not. But the antibodies are developed late in the stage of infection so the test is used to check the prevalence of the disease in a particular area.

The RT-PCR test is considered as the highest standard test for diagnosing the COVID-19 test which includes testing of nasal swabs from mouth and nose. Although the accuracy of the test varies between 70 to 80 percent it the most reliable test which doctors rely upon in order to treat for COVID-19. The error is mainly due to improperly collecting the samples, manhandling of the samples, interchanging of the samples which either shows false results sometimes or shows inconclusive result where in both the cases re-test is done. Before admitting the pregnant women to the hospital, it is now mandatory to test her for COVID-19 to avoid further complications. Although if the test resultstake time the hospital administration must admit the patient and treat it like the result is positive in order to be careful for any scenario.

Although the percentage of the pregnant women with non-pregnant women is at par in terms of contracting the disease which stands at 9.3 and 11 percent with milder symptoms and 2 and 7 percent with severe symptoms (Cheng et al., 2020), the severity shown by the disease in pregnant women may change with respect to general population. Although more comprehensive data is needed to further establish the correlation. The maternal mortality rate (MMR) is not distinctly different in COVID19 than in previous similar yet small scale outbreaks of Severe Acute respiratory syndrome (SARS) and Middle Eastern respiratory syndrome (MERS). Though they have to offer some lessons which can be implemented in dealing with containment of the COVID-19. But the risk is high as they are exposed to doctors and patients dealing with COVID-19 so complacency should not be practiced. Increased monitoring and regular testing even after getting the first test negative mist be dome in order to secure and reduce the case fatality rate (CFR) and severity of the symptoms and make neonates safer.

Covid-19 and Child Births: New born babies or infants are most vulnerable especially in their initial forming years. Prior to that if a pregnant woman got infected with COVID-19 in either of the three trimesters the risk of various child or infant related complications and severe outcomes may increase like still birth, maternal deaths etc. A study conducted by world association of prenatal medicine (WAPM) which covers 22 countries have lot results emanating from their study. The study involved 388 pregnant cases with infection of COVID19. According to the study 11 percent of the studied women was shifted to ICU while three of them died making maternal mortality rate at 0.8 percent (Huntley et al., 2020). There were 70 preterm birth but it largely includes voluntary requests from patients to prepone the delivery to avoid any COVID-19 complications. Few of them were non-voluntary. 2.3 percent cases saw 
miscarriages which stands at the figure 6 and 5 neo natal deaths. The symptoms recorded mostly in another study which includes patients from China, USA amounting to 108 were fever, persistent dry cough, and shortness of breath. Less commonly symptoms include dyspnea, diarrhea, hypoxia etc.

In another study 7 out of 9 pregnant women died possibly due to COVID-19 complications, other two being either in ICU or on ventilator support. Mostly they are in last part of the second trimester and third trimester of their pregnancy. In some cases, obesity as comorbidity worsened the patient's condition and it was difficult to overcome from the situation for the patient. The vast difference in the maternal mortality rate throughout the globe. As the study conducted in United States of America about forecasting the death and severity rate among the maternity ward patients, it estimated around 3300 severe cases and around 600 critical cases to be arise from March 2020 to the end of this year. Also, it also unfortunately predicted on the basis of the current available data, 52 COVID-19 related deaths due to complications is predicted using mathematical modelling. It indicates the grave threat that COVID-19 pandemic poses to maternity patients (Hantoushzadeh et al., 2020).

Vertical Transmission: Vertical transmission of the COVID-19 is the highly debated phenomenon which is not proven yet but complacency has no place as far as infants are concerned. Vertical transmission(10) is the case in which the coronavirus disease 2019 is transmitted or passed on by mother to her offspring. But this fact is highly debated and not yet established by empirical evidences. Though some of the infants tested positive for the disease, they are not attributed to the vertical transmission case. The neonates who tested positives were showing mild to none symptoms in most of the cases. The amnioticfluid, placenta samples were tested negative which also ruled out the case of vertical transmission. The reason behind the infants contracting lies in external environment in which it was exposed. Even breast milk was tested negative so there was no transmission through breastfeeding which is most essential part of infant nutrition and its immunity.

Although while handling baby by positive mother and possibility of sneezing or coughing near baby might have the carrier role to transfer the infection. Although vertical transmission is not completely ruled out yet and should be watched after in future course. The delivery mechanism for the child is also the deciding factor whether the chances of the infection is high or not. Normal or vaginal deliveries are overpowered by cesarean section deliveries. The later has higher chances of the transmission of the disease as compared to former one.Aerosol transmission containing the coronavirus in short range can be the possible reason behind the phenomenon( Kotlyar et al., 2020). Testing samples of placenta, blood, amniotic fluid is necessary to establish any correlation between the two.
Non-pharmacological interventions (NPI's) like wearing masks and hand gloves by infected mother when handling infants must be followed in order to safeguard the baby from the infection. Also exposing the infant to the infected mother only when necessary activity needs to be carried out can drastically reduce the chances of the transmission of infection. The neonatal result and the condition of the infant is highlydependent on the mother's condition and the time of delivery. The pre term delivery is onof the factor behind some deaths reported due to neonatal infection of the COVID-19. Although there is no established study but infants delivered after full 36 weeks gestational period are far less prone to showing severe symptoms.

Post-natal care also plays aninfluential role and the environment, health care facility, following of the guidelines both by the relatives and the doctors and allied health care professionals are some factors that can seriously affects the outcome in COVID-19 pandemic with respect to maternity ward is concerned. The concerning factor is the authenticity of the data. As the data is gathered by multiple agencies and wrong attribution cannot be ruled out. The homogeneity of the data and results of the samples collected and wide variation in treatment protocols may be an obstacle in arriving at a conclusion. But making repository more robust and authentic can be a solution so as to include in the containment strategy of the COVID-19 (Fenizia et al., 2020).

Psychological Impact of the Covid-19 On Pregnant Women: The COVID-19 not only affects the physical and anatomical aspects of the affected person but also mental aspects as huge amount of uncertainty is involved due to newness of the disease. Scientists and researchers have no data of previous such occurrences as the COVID-19 is novel pandemic. Especially when the case fatality rate is touching extremely high marks as compared to other widespread diseases and there is no sign of vaccine or preventive therapy the mental distress (Balkhi et al., 2020)is surely scaling high. Despite the social distress cuts across all sections of the society, pregnant women are more vulnerable than others. Already the hormonal changes brought about by pregnancy can severely affects the mood and psyche of the mother and which needs to be looked upon, the case for the same worsens in times of global distress like COVID-19.

The fear of contracting the virus is on top but passing it on to their offspring paramount all others. The daily news of the worsening condition due to COVID-19 and uncertainty (Dubey et al., 2020)about wellbeing of theirs and their infants constantly haunts them and can create severe psychological distress which is especially bad in pregnant state. The crumbling health care infrastructure gives no assurance to the patient that he will be accommodatedwithout getting the infection. Even in some cases deliveries of the babies were happing through local midwifes to avoid any contact with the clinics which are harboring the COVID-19 patients. It is a concerning fact that non-institutionaldelivering of the 
infants is on rise due to inappropriate facilities, lack of hygiene and competence. It can create another dictator if this trend lives on.

Widespread misinformation and fake news about hospitals being falsely giving positive results of the patients in order to cash in more money can prevent anyone from the hospitals if believed. The home remedies which are not certified by any authority and keeps on circulating the social networking sites can prove fatal if proper contraindications are not known which is maximum cases are not known. First ofall, the deliveries should be carried out in recognized institutions or through registered midwifes in order to reduce maternal mortality rate (MMR)and infant mortality rate (IMR). A confidence building campaign with assuring all the help in worst case scenario can win back the lost trust of the people. Disbursing authentic and verified information without any time lag as misinformation spreads like wild fire must be done in order to contain the Infodemic that is gaining some ground and found some buyers.

Post-Covid-19 Care In Pregnant Women: COVID-19 not only affects while infected, it needs proper monitoring post-COVID19 too. Studies suggests that long term implications are yet to surface and persistence of the symptoms have been found in some cases. Researchers termed it as long COVID-19 condition(15). Some people who tested positive and undergone treatment have experiencing certain set of symptoms even after officially recovering from the infection. This includes persistence fatigue, sleep apnea, loss of taste and smell, cough even after they got the virus way back in march and April of 2020. This persistence of symptoms in some individuals is also known as COVID-19. The severity of long can't be measures on case fatality rate scale but it is seen that healthy athletic persons lost their ability to execute as vigorously as they were doing. Some people required inhalers which they hadn't used in their life prior to COVID-19. This is an extremely fresh phenomenon and needs comprehensive study.

As some countries planning to ride on her immunity tide. By looking at long COVID-19 condition it would definitely hamper the prospect of these agencies. Recurrence of one symptom or the other is a signature mark of the long COVID-19 as far as this time is concern. This has created the need of study of long-term impact study on very large people to conclude or correlate the data. Careless people who generally defies the guidelines and rule made or they so that they are safe from the contraction are more prone to such consequences. More importantly men behave less responsibly than women, if the long COVID-19 conditions find the ground among larger masses it will be again more deadly for than women as the case is now. This might affects the maternal and child mortality rates which needs to be controlled according various global initiatives like sustainable development goals which are pending to achieve by 2030 (Khoiwal et al., 2020).
A number of related articles were reviewed (Gaidhane et al., 2020; Jachak et al., 2020; Joseph et al., 2020; Lakhkar et. al., 2020) outlined the sufferings of pregnant women in Covid-19 (Ghate et. al., 2020; Mujbaile et. al., 2020) also reported similar studies on sufferings of pregnant women in COVID-19.Studies by (Arriola et.al., 2020; Karadbhajane et.al., 2020; Mude et.al., 2020) were reviewed. Key aspects of therapeutic issues and problems in Covid-19 were reviewed.

\section{CONCLUSION}

The news about rolling out of the vaccine for the COVID19 can cheer up the society to some extent but the reaction and acceptability by the body is yet to be seen. Therefore, still a considerable amount of time is left to take care of the infected persons particularly pregnant women in this case is of utmost importance. Some of the healthcare facilities can converted to exclusively treat the pregnant women and treatment of the neonates in order to safeguard them from the disaster of COVID19. This will bring down the unsafe practice of noninstitutional deliveries which are being carried due to the fear of contracting the virus. Vertical transmission needs more study and data analyzation then one can treat according to the correlation of the two. However preventable transmission of the COVID-19 infection by wearing masks and gloves and isolating the baby whenever possible from the infected mother should be carried out.

Psychological distress can be addressed in pregnant women by enabling them to meet their loved ones with proper safety measures in order to relive their anxiety. Dedicated psychologists and community doctors can be employed in every facility to counsel the patient and their relative. As far as Infodemic is concern, counter misinformation warfare needs to be activated by the governmental agencies to demystify the false information which in some cases may prove fatal and consumes lots of resources. Persistence of the symptoms called long COVID-19 condition is yet another challenge and as the pandemic is still unfolding all the trends needs to be carefully monitored. Vulnerable groups such as pregnant women and neonates needs special attention to overcome the trauma physically as well as mentally to lead a happy life.

\section{REFERENCES}

Arriola, Carmen S., piyaratsuntarattiwong, Fatimah S. Dawood, Giselle Soto, Prabir Das, Danielle R. Hunt, chalinthornsinthuwattanawibool, et al. 2020 "What Do Pregnant Women Think about Influenza Disease and Vaccination Practices in Selected Countries." HUMAN VACCINES \& IMMUNOTHERAPEUTICS, n.d. Https://doi. org/10.1080/21645515.2020.1851536.

Azer SA. 2020 COVID-19: pathophysiology, diagnosis, complications and investigational therapeutics. New Microbes and New Infections, 37:100738. 
Balkhi F, Nasir A, Zehra A, Riaz R. Psychological and Behavioral Response to the Coronavirus (COVID19) Pandemic. Cureus https://www.cureus.com/ articles/31114-psychological-and-behavioral-responseto-the-coronavirus-covid-19-pandemic

Cheng S0, Khan S, Alsafi Z. 2020 Maternal death in pregnancy due to COVID-19. Ultrasound Obstet Gynecol.56(1):122-122.

Di Mascio D, Sen C, Saccone G, Galindo A, Grünebaum A, Yoshimatsu J, et al. 2020 Risk factors associated with adverse fetal outcomes in pregnancies affected by Coronavirus disease 2019 (COVID-19): a secondary analysis of the WAPM study on COVID-19. Journal of Perinatal Medicine. 48(9):950-8.

Dubey S, Biswas P, Ghosh R, Chatterjee S, Dubey MJ, Chatterjee S, et al. 2020 Psychosocial impact of COVID19. Diabetes \& Metabolic Syndrome: Clinical Research \& Reviews.14(5):779-88.

Dushyant Bawiskar, Pratik Phansopkar, Ayurva Vilas Gotmare. 2020 COVID-19 Facets: Pandemics, Curse and Humanity. IJRPS, 6;11(SPL1):385-90.

Facchetti F, Bugatti M, Drera E, Tripodo C, Sartori E, Cancila V, et al. 2020 SARS-cov2 vertical transmission with adverse effects on the newborn revealed through integrated immunohistochemical, electron microscopy and molecular analyses of Placenta. Ebiomedicine https://www.thelancet.com/journals/ebiom/article/ PIIS2352-3964(20)30327-3/abstract

Fenizia C, Biasin M, Cetin I, Vergani P, Mileto D, Spinillo A, et al. 2020 nalysis of SARS-cov-2 vertical transmission during pregnancy. Nature Communications. 11(1):5128.

Gaidhane S, Khatib N, Zahiruddin QS, Gaidhane A, Telrandhe S, Godhiwal P. 2020 'Depression, anxiety and stress among the general population in the time of COVID-19 lockdown: A cross-sectional study protocol.' International Journal of Research in Pharmaceutical Sciences, 11(1), pp. 360-364.

Gawai, Jaya Pranoykumar, Seema Singh, Vaishali deoraojitaksande, Tessy Sebastian, Pooja Kasturkar, and Ruchira Shrikant Ankar. 2020 "Critical Review on Impact of COVID 19 and Mental Health.” JOURNAL OF EVOLUTION OF MEDICAL AND DENTAL SCIENCESJEMDS 9: 2158-63. Https://doi.org/10.14260/ jemds/2020/470.

Ghate, V.C., S. Borage, and P. Shelotkar. 2020 “Covid-19 in Pregnant Women." International Journal of Research in Pharmaceutical Sciences 11: 430-32. Https://doi. org/10.26452/ijrps.v11ispl1.2805.

Hantoushzadeh S, Shamshirsaz AA, Aleyasin A, Seferovic MD, Aski SK, Arian SE, et al. 2020 Maternal death due to COVID-19. American Journal of Obstetrics and Gynecology. 223(1):109.e1-109.e16.
Huntley BJF, Huntley ES, Di Mascio D, Chen T, Berghella V, Chauhan SP. 2020 Rates of Maternal and Perinatal Mortality and Vertical Transmission in Pregnancies Complicated by Severe Acute Respiratory Syndrome Coronavirus 2 (SARS-Co-V-2) Infection: A Systematic Review. Obstetrics \& Gynecology. 136(2):303-12.

Jachak, S., P. Phansopkar, and M. Waqar Naqvi. 2020 "Impact of Covid-19 in India, a Disastrous Pandemic Outbreak." International Journal of Research in Pharmaceutical Sciences 11:399-402. Https://doi. org/10.26452/ijrps.v11ispl1.2735.

Joseph, M.B., S. Pohekar, A. Raut, and M. Patil. 2020 "The Palliative Care and Covid-19 Pandemic." International Journal of Research in Pharmaceutical Sciences 11:618-22. Https://doi.org/10.26452/ijrps. v11ispl1.2861.

Joshi, K., N. Acharya, S. Acharya, and S. Joshi. 2020 "A Grave Situation with COVID in the Gravid: A Narrative Review." International Journal of Research in Pharmaceutical Sciences 11:496-99. Https://doi. org/10.26452/ijrps.v11ispl1.2837.

Karadbhajne, Priti, Anil Tambekar, Abhay Gaidhane, Zahiruddin Quazi Syed, Shilpa Gaidhane, and Manoj Patil. 2020 "Amoebiasis in Pregnant Woman: A Case Report.” MEDICAL SCIENCE 24: 1814-17.

Khatib, M.N., S. Gaidhane, M. Khatib, M. Ahmed, A. Gaidhane, and Z.Q. Syed. 2020 "SARS-cov and SARScov-2: Similar Viruses with Different Trajectories.” Wutan Huatan Jisuan Jishu 16:544-48.

Khoiwal K, Kapur D, Gaurav A, Chaturvedi J. 2020 Management of Pregnant Women in Times of Covid19: A Review of Current Literature. J Obstet Gynaecol India. 7;1-5.

Khubchandani, Sheetal Rameshlal, and Trupti Madhav Dahane. 2020 "Emerging Therapeutic Options for COVID-19.” JOURNAL OF EVOLUTION OF MEDICAL AND DENTAL SCIENCES-JEMDS 9: 3082-85. Https:// doi.org/10.14260/jemds/2020/677.

Kotlyar AM, Grechukhina 0, Chen A, Popkhadze S, Grimshaw A, Tal 0, et al. 2020 Vertical transmission of coronavirus disease 2019: a systematic review and meta-analysis. American Journal of Obstetrics and Gynecology http://www.sciencedirect.com/science/ article/pii/S0002937820308231

Kute, Vivek, Sandeep Guleria, Jai Prakash, Sunil Shroff, Narayan Prasad, Sanjay K. Agarwal, Santosh Varughese, et al. 2020 "NOTTO Transplant Specific Guidelines with Reference to COVID-19." INDIAN JOURNAL OF NEPHROLOGY 30: 215-20. Https://doi.org/10.4103/ijn. IJN_299_20.

Lakhkar, B.B., B. Guru, S. Damke, and S. Damke. 2020 "Most Susceptible Duo in COVID-19 Crisis: A Literature Review.” Perinatology 21: 112-23. 
Latchoumi, T.P., Ezhilarasi, T.P. and Balamurugan, K., 2019. Bio-inspired weighed quantum particle swarm optimization and smooth support vector machine ensembles for identification of abnormalities in medical data. SN Applied Sciences, 1(10), pp.1-10.

Mudey, D. A., D. M. Khapre, D. G. Mudey, and D. R. Goyal. 2015 “An Innovative Approach of Mobile E-Health Intervention in Tracking Antenatal Mothers \& Neonates in Selected Rural Areas of a District in Central India: An Ice Breaking Footstep for Revolution." INTERNATIONAL JOURNAL OF EPIDEMIOLOGY 44: 95-96.

Mujbaile, N.S., and S. Damke. "The Impact of COVID 19 on Pregnant Women and Child Health.” International Journal of Research in Pharmaceutical Sciences 11, no. Special Issue 1 (2020): 1367-73. Https://doi. org/10.26452/ijrps.v11ispl1.3645.

Qiancheng X, Jian S, Lingling P, Lei H, Xiaogan J, Weihua L, et al. 2020 Coronavirus disease 2019 in pregnancy. International Journal of Infectious Diseases. ;95:376-83.

Sheriff D. 2020 Health care in India in the prevailing COVID-19 pandemic scenario. Eubios journal of Asian and international bioethics: EJAIB. 19;30:91.

Wang B, Li R, Lu Z, Huang Y. 2020 Does comorbidity increase the risk of patients with COVID-19: evidence from meta-analysis. Aging (Albany NY). 8;12(7):604957.

WHO Director-General's opening remarks at the media briefing on COVID-19 - 11 March 2020: https://www. who.int/director-general/speeches/detail/who-directorgeneral-s-opening-remarks-at-the-media-briefing-oncovid-19---11-march-2020 